of control group $(-2.07 \pm 0.62 \mathrm{~s}$ vs $-2.33 \pm 0.57 \mathrm{~s}, \mathrm{p}<0.05)$. SrCa and $\mathrm{SrRa}$ had no significant differences between any two groups.

Conclusions Left ventricular diastolic longitudinal and circumferential functions were significantly lower in T2DM patients with normal LVEF and without symptomatic heart failure. And the T2DM patients combined with HT had impaired left ventricular diastolic function of longitudinal, circumferential and radial directions, so they had even more severe left ventricular diastolic dysfunction. STI could evaluate subclinical left ventricular diastolic dysfunction in longitudinal, circumferential and radial directions, and early diastolic strain rate in every direction could evaluate left ventricular diastolic function with high sensitivity.

\section{GW23-e1284 EARLY DETECTION OF LEFT VENTRICULAR DIASTOLIC DYSFUNCTION IN PATIENTS WITH TYPE 2 DIABETES MELLITUS BY SPECKLE TRACKING IMAGING}

doi:10.1136/heartjnl-2012-302920o.13

Fan Rui, Lin Hong. Department of Ultrasound, First Affiliated Hosptial, Zhongshan University

Objectives The purpose of the study was to assess subclinical left ventricular longitudinal, circumferential and radial diastolic dysfunction in patients with T2DM or combined with HT by STI.

Methods 77 T2DM patients with normal LVEF and without symptomatic heart failure were divided into two groups according to their blood pressure. Patients with T2DM isolated (DM): 39 cases (22 men, 17 women, mean age $49.62 \pm 9.35$ years), and associated with HT (DH): 38 cases (20 men, 18 women, mean age 51.39 \pm 9.19 years). Control group (Normal): 34 healthy individuals (16 men, 18 women, mean age $49.68 \pm 10.74$ years). All subjects were underwent transthoracic echocardiography. Global early diastolic peak longitudinal strain rate (SrLe) and late diastolic peak longitudinal strain rate (SrLa) were measured by SRI and STI. Also, we measured early diastolic peak circumferential strain rate $(\mathrm{SrCe})$, late diastolic peak circumferential strain rate $(\mathrm{SrCa})$, early diastolic peak radial strain rate $(\mathrm{SrRe})$ and late diastolic peak radial strain rate $(\mathrm{SrRa})$ by STI. Multiple comparisons were performed using ANOVA with post hoc Dennett's or Student-Newman-Keuls test. Statistical significance was inferred for $\mathrm{p}<0.05$.

Results Global SrLe derived from SRI were significantly lower in the two patient groups (DM: $1.20 \pm 0.30 \mathrm{~s} ; \mathrm{DH}: 1.00 \pm 0.28 \mathrm{~s}$ ) than that of control group $(1.75 \pm 0.46 \mathrm{~s})$ and significant differences were observed between any two groups $(p<0.05)$, and global SrLe derived from STI were also significantly lower in the two patient groups (DM: $1.47 \pm 0.25 \mathrm{~s}$; DH: $1.31 \pm 0.26 \mathrm{~s}$ ) than that of control group $(2.08 \pm 0.34 \mathrm{~s})$ and significant differences were observed between any two groups $(p<0.05)$, while SrLa derived from SRI and STI had no significant differences between any two groups. Global SrCe derived from STI was significantly lower in the two patient groups (DM: $1.73 \pm 0.33 \mathrm{~s}$; DH: $1.59 \pm 0.30 \mathrm{~s}$ ) than that of control group $(2.10 \pm 0.33 \mathrm{~s})$ and significant differences were observed between patient groups and normal group $(p<0.05)$. Global SrRe were only significantly lower in $\mathrm{DH}$ group than that 